\title{
Nonlinear circuit design of damping boring bar based on MULTISIM
}

\author{
HAO Ruican ${ }^{1 \mathrm{a}}$, Gu Chunguang ${ }^{1}$, Wang Rongdong ${ }^{2}$ and Zhang $\mathrm{Na}^{1}$ \\ ${ }^{1}$ School of Mechanical Engineering, Beijing Polytechnic, Beijing, China \\ ${ }^{2}$ Beijing Feb.7th Railway Transportation Equipment Co., Ltd, Beijing, China \\ ahaoruican@163.com
}

Keywords: damping boring bar; nonlinear; MATLAB; MULTISIM; circuit design

\begin{abstract}
Chatter easily exits during cutting process of damping boring bar. In order to save development cycle of damping boring bar circuit, nonlinear model of damping boring bar is established with help of SIMULINK model of MATLAB. Modular layout of corresponding arithmetic logic unit is designed under modular design. Layout of arithmetic logic unit contributes to design of circuit. The circuit of system is designed and simulated with MULTISIM. The development of circuit contributes to research on control strategy concerning chatter. It also provides a new approach to pulling progress of nonlinear dynamics.
\end{abstract}

\section{Introduction}

Precision cutting is one vital means about machining deep holes. However, during cutting period, low rigidity of damping boring bar leads to vibration thus influencing quality of machining. Experts from home and overseas carry on responding research on cause of vibration and control strategy. R.S.Hahn[1] regards that regenerative chatter is caused by regenerative effect of ripples when machining. However, research on analogical circuit of damping boring bar is rare, so is universal device contributing to research on dynamics. Plotting of circuit diagram of damping boring bar contributes to research on design and control strategy of damping boring bar thus pushing progress of nonlinear dynamics forward.

\section{Damping boring bar mathematical model}

Selection damping block length $120 \mathrm{~mm}$, with a radius of $10 \mathrm{~mm}$ of lead material, can calculate the mass $m_{2}=0.426 \mathrm{~kg}$, other material parameters: $k_{1}=9.44 \times 10^{5}, k_{2}=1.31 \times 10^{5}, c_{2}=70.18$. Kinetic model shown in Fig.1.

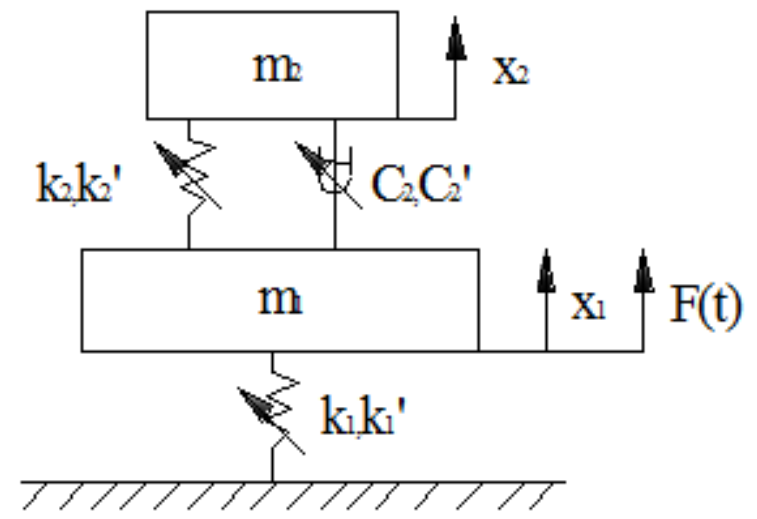

Fig. 1 The dynamic model of damping boring bar

First, set the system of nonlinear elastic force equation:

$$
f_{1}\left(x_{1}\right)=k_{1} x_{1}+k_{1}^{\prime} x_{1}^{3} \quad f_{2}\left(x_{2}\right)=k_{2}\left(x_{2}-x_{1}\right)+k_{2}^{\prime}\left(x_{2}-x_{1}\right)^{3}
$$

Set of nonlinear damping force system of equations:

$$
R_{2}\left(x_{2}\right)=c_{2}\left(\dot{x}_{2}-\dot{x}_{1}\right)+c_{2}^{\prime}\left(x_{2}-x_{1}\right)^{2}\left(\dot{x}_{2}-\dot{x}_{1}\right)
$$

Differential equations of motion for the system: 


$$
\begin{aligned}
& m_{1} \ddot{x}_{1}+k_{1} x_{1}+k_{1}^{\prime} x_{1}^{3}-k_{2}\left(x_{2}-x_{1}\right)-k_{2}{ }^{\prime}\left(x_{2}-x_{1}\right)^{3}-c_{2}\left(\dot{x}_{2}-\dot{x}_{1}\right)-c_{2}{ }^{\prime}\left(x_{2}-x_{1}\right)^{2}\left(\dot{x}_{2}-\dot{x}_{1}\right)=F(t) \\
& m_{2} \ddot{x}_{2}+k_{2}\left(x_{2}-x_{1}\right)+k_{2}{ }^{\prime}\left(x_{2}-x_{1}\right)^{3}+c_{2}\left(\dot{x}_{2}-\dot{x}_{1}\right)+c_{2}{ }^{\prime}\left(x_{2}-x_{1}\right)^{2}\left(\dot{x}_{2}-\dot{x}_{1}\right)=0
\end{aligned}
$$

Due to the displacement of the damping blocks affected by the inner diameter of the boring bar, more importantly, the damping block with respect to the displacement of the boring bar. The relative displacement of damping blocks into equation (3) can be obtained

$$
\begin{aligned}
& \left(m_{1}+m_{2}\right) \ddot{x}_{1}+m_{2} \ddot{x}_{21}+k_{1} x_{1}+k_{1}^{\prime} x_{1}^{3}=F(t) \\
& m_{2} \ddot{x}_{1}+m_{2} \ddot{x}_{21}+k_{2} x_{21}+k_{2}{ }^{\prime} x_{21}^{3}+c_{2} \dot{x}_{21}+c_{2}{ }^{\prime} x_{21}^{2} \dot{x}_{21}=0
\end{aligned}
$$

\section{MATLAB/SIMULINK Simulation}

MATLAB / SIMULINK is an integrated software package for modeling and simulation of dynamic systems and analysis, it can invoke powerful MATLAB database. It is very comprehensive analysis capabilities can handle linear and non-linear, discrete and continuous systems. And supports different rate of change in the same system, so for the boring bar vibration analysis using simulation software MATLAB / SIMULINK. (4), established according to the dynamic damping boring bar system of differential equations which SIMULINK simulation model.Open MATLAB simulation switch, main system phase diagram shown in Fig.2.

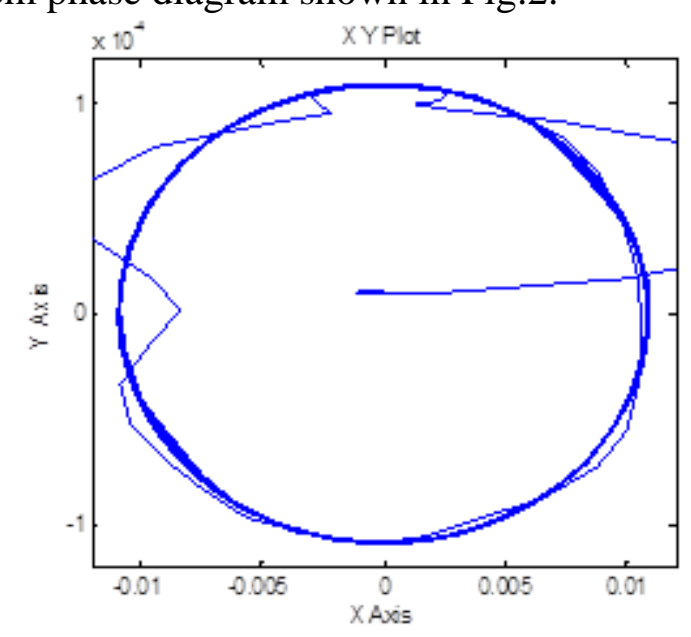

Fig.2 The phase diagram of main system

\section{MULTISIM Simulation}

Since the input resistance $\mathrm{Ri}$ inverting integrator circuit $=\mathrm{R} 1$, the input resistance of the amplifier in terms of the bigger the better, so the better the resistance R1. When the input resistance meets the system requirements, the capacitor selection is generally not worth more than $1 \mu \mathrm{F}$. Resistor R2 is a static balancing resistor, whose role is used to compensate for offset bias current generated, and generally R2 = R1. MULTISIM boring bar vibration simulation of nonlinear systems is shown in Fig.3. 


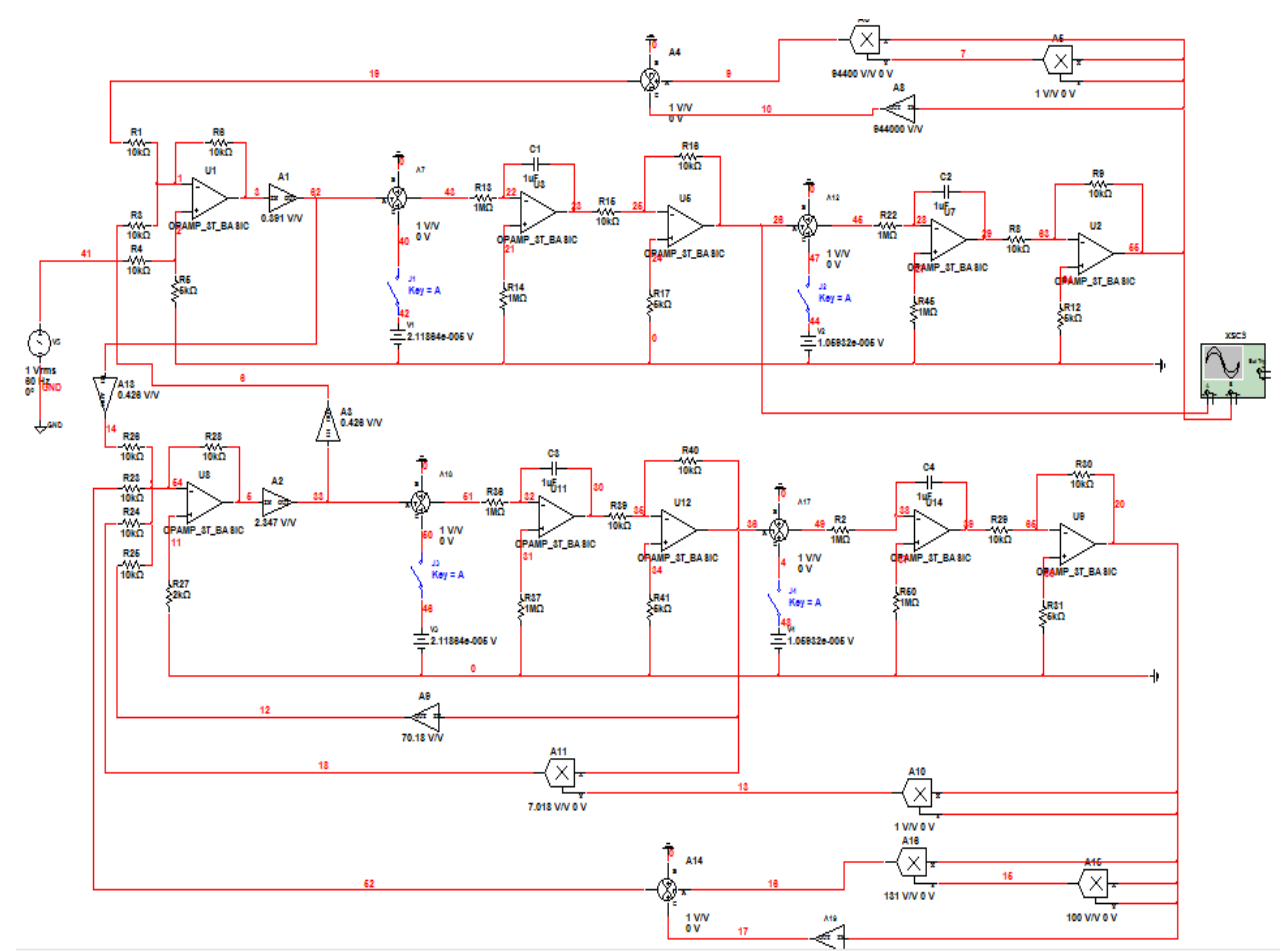

Fig.3 The circuit schematic simulation of damping boring bar with MULTISIM

Circuit design and simulation steps: (1) According to kinetic model building MATLAB / SIMULINK simulation platform built in the corresponding circuit; (2) in accordance with specific parameters editing component design requirements; (3) according to the testing requirements, connect the appropriate virtual instrumentation; (4) the circuit design and simulation, the simulation results were observed; (5) to modify the component parameters, observations change, system optimization design ${ }^{[2,3]}$. Circuit schematic design of the nonlinear damping boring bar system is divided into two parts: the main system and shock absorber. The first is the integration circuit design system, provide the basis for building relationships after the function. Circuit diagram U3, U7, U11, U14 for integrating circuit module. Plus the integrated signal, initial and ground signals ${ }^{[4]}$ A7, A12, A17, A18 is to provide the initial value of the module, the adder circuit for integrating the three terminals, respectively. Since the contrast of the output signal of the system integration module of the input signal symbol, and therefore need to add a variable symbol module (i.e. inversely proportional to the module, a coefficient of -1) U2, U5, U9, U12 sign of the signal to be consistent. U1, U8 for addition and subtraction circuit, complete the combined system function relationships. A1, A2, A8, A9 gain modules, the system provides proportional coefficient, the ratio of the specific module may be replaced. A5, A6, A10, A11, A15, A16 as a multiplier, the system provides a nonlinear operation. Open the simulation switch, phase diagram of the main system as shown in Fig.4.

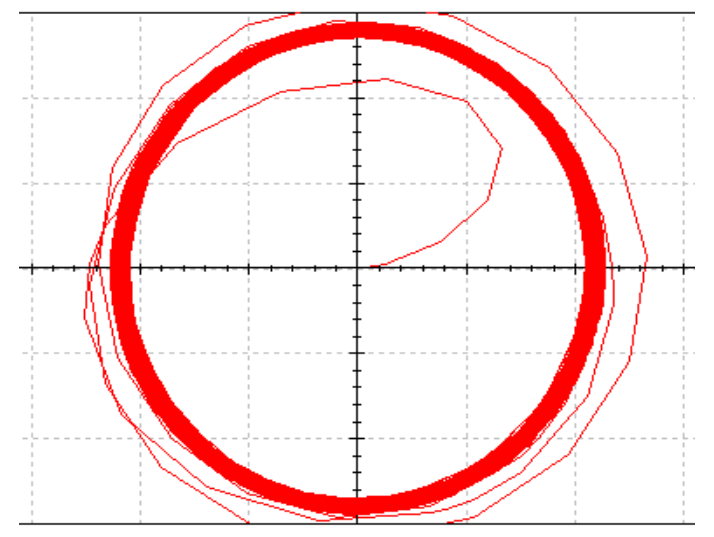

Fig.4 phase diagram of main system based on MULTISIM 


\section{Conclusion}

According to dynamic math model of nonlinear damping boring bar, Simulink model of system is constructed with modeled construction. Models in Matlab are replaced by corresponding circuit model with EDA software Multisim. Through combination of models, circuit diagram of system is modeled. From simulation, we can conclude that through simulation of Simulink and Multisim, we can see that phase diagram of main system is same thus proving correctness of nonlinear circuit of damping boring bar.

\section{Acknowledgements}

This project is supported by Beijing Municipal Commission of Education Technology Plan plane project(KM201410858001), and Beijing Polytechnic Science and Technology Project (YZK2015017) .

\section{References}

[1] Dj.M. Maric, P.F. Meier and S.K. Estreicher: Mater. Sci. Forum Vol. 83-87 (1992), p. 119

[2] M.A. Green: High Efficiency Silicon Solar Cells (Trans Tech Publications, Switzerland 1987).

[3] Y. Mishing, in: Diffusion Processes in Advanced Technological Materials, edtied by D. Gupta Noyes Publications/William Andrew Publising, Norwich, NY (2004), in press.

[4] G. Henkelman, G.Johannesson and H. Jónsson, in: Theoretical Methods in Condencsed Phase Chemistry, edited by S.D. Schwartz, volume 5 of Progress in Theoretical Chemistry and Physics, chapter, 10, Kluwer Academic Publishers (2000).

[5] R.J. Ong, J.T. Dawley and P.G. Clem: submitted to Journal of Materials Research (2003)

[6] P.G. Clem, M. Rodriguez, J.A. Voigt and C.S. Ashley, U.S. Patent 6,231,666. (2001)

[7] Information on http://www.weld.labs.gov.cn 